\title{
Plasma Plume of Annular and Cylindrical Hall Thrusters
}

\author{
N. J. Fisch and Y. Raitses
}

\begin{abstract}
Hall thrusters hold considerable advantage over chemical thrusters and other kinds of electrical propulsion devices, except that the plume of the Hall thruster tends to be wide. An ongoing objective in Hall-thruster research is to narrow this plume. The plume is sensitive both to the specific geometry of the magnetic field as well as to the voltage potential induced within the plasma. Annular-geometry Hall thrusters tend to have narrower plumes. However, the cylindrical-geometry thruster has been shown to be suited particularly to low-power operation. New techniques have been advanced to narrow the Hall-thruster plumes. In this paper, images of the plasma plume in the two geometries of the Hall thruster are presented.
\end{abstract}

Index Terms-Hall thruster, plasma plume.

$\mathbf{P}$ LASMA thrusters are used to compensate for atmospheric drag on satellites in low-Earth orbit, to reposition satellites in geosynchronous orbit, or to raise a satellite from a lower orbit to geosynchronous orbit. The advantage of plasma thrusters over conventional chemical thrusters is that the exhaust energies are not limited by chemical energy to on the order of an electron volt. Instead, exhaust energies in plasma thrusters are limited by the available onboard power and can reach hundreds of electron volts, thereby requiring less onboard mass. For each kilogram of satellite mass, about $1-3 \mathrm{~W}$ of onboard power tend to be available.

A Hall thruster [1] overcomes the space-charge limit of the ion thruster. An ion thruster consists of an anode and a cathode, between which a voltage drop occurs essentially in vacuum. In a Hall thruster, the intervening space is filled with plasma. The plasma neutralizes the space charge that would otherwise limit the flow of ions in the vacuum. However, to retain the electrons in the flow, that is to prevent the electrons from flowing too swiftly toward the anode, in a Hall thruster, a radial magnetic field is introduced. The magnitude of the field is approximately $200 \mathrm{G}$, strong enough to prevent the axial flow of electrons, causing them instead to drift azimuthally. This configuration, therefore, is necessarily annular in design, since the magnetic-field pole must be predominantly radial throughout the acceleration region. Another feature of the Hall thruster is that taking the place of the gridded cathode of the ion thruster is an electron emitter, or plasma hollow-cathode

Manuscript received December 17, 2007; revised March 4, 2008. This work was supported by the U.S. DOE under Contract AC02-76CH0-3073.

The authors are with the Princeton Plasma Physics Laboratory, Princeton University, Princeton, NJ 08543-0451 USA (e-mail: fisch@pppl.gov; yraitses@ pppl.gov).

Digital Object Identifier 10.1109/TPS.2008.925696 neutralizer. Through electron emission from the cathode, the hollow-cathode neutralizer establishes a virtual cathode within the plasma flow from the thruster.

Plasma thrusters for current space applications employ xenon propellant. Xenon is relatively easy to ionize and store onboard the spacecraft. It also has a high atomic number (54), which means more mass per ionization energy expended. The ionization energy is a necessary inefficiency; in the range of exhaust velocities most useful for current space applications-about $15-20 \mathrm{~km} / \mathrm{s}$ - this energy loss for once-ionized xenon is less than $10 \%$ of the exhaust energy. For many missions, higher exhaust velocities tend to require too much power for the same thrust, and lower exhaust velocities tend to require too much mass.

In the annular Hall thruster, the electron axial motion is fully impeded, at least in the absence of collisions, by the radial magnetic field. The axial electron current that does flow represents an additional inefficiency, due to the power dissipated by the electron current. The cylindrical Hall thruster [2] removes the restriction that electrons cannot travel axially and, instead, restricts only that, on average, the electrons, in the absence of collisions, carry no axial current, and therefore, no current losses. This is accomplished by having a magnetoelectric trap, whereby the magnetic-field lines diverge between the anode and the cathode, such that the electrons are magnetically reflected near the anode but electrostatically reflected near the cathode.The ions, as in the annular case, are not magnetized.

One of the key issues, however, in both the annular and the cylindrical thruster is the divergence of the plasma plume. In the case of the annular thruster, attempts have been made to narrow the plume through the introduction of segmented electrodes along the thruster channel [3], [4]. The control of the plume in the annular thruster might occur through the control of this potential [5], [6] or through a magnetic-lens effect [1], [7]. In Fig. 1(a), we show the plasma plume from an annular thruster.

The plume of the cylindrical thruster [1], [8] is naturally broader than the plume of the annular thruster. However, recently, it was shown that, in certain regimes of increased cathode current, it was possible to narrow this plume considerably [9]. The physics of the plume narrowing of the cylindrical thruster is a subject of ongoing investigation. In Fig. 1(b), we show an image of the plume from a cylindrical thruster.

In conclusion, images of Hall-thruster plasma plumes have been presented. The cylindrical Hall thruster naturally has a wider plume, but methods have been advanced to narrow the plasma plume considerably on both kinds of plasma thrusters. 

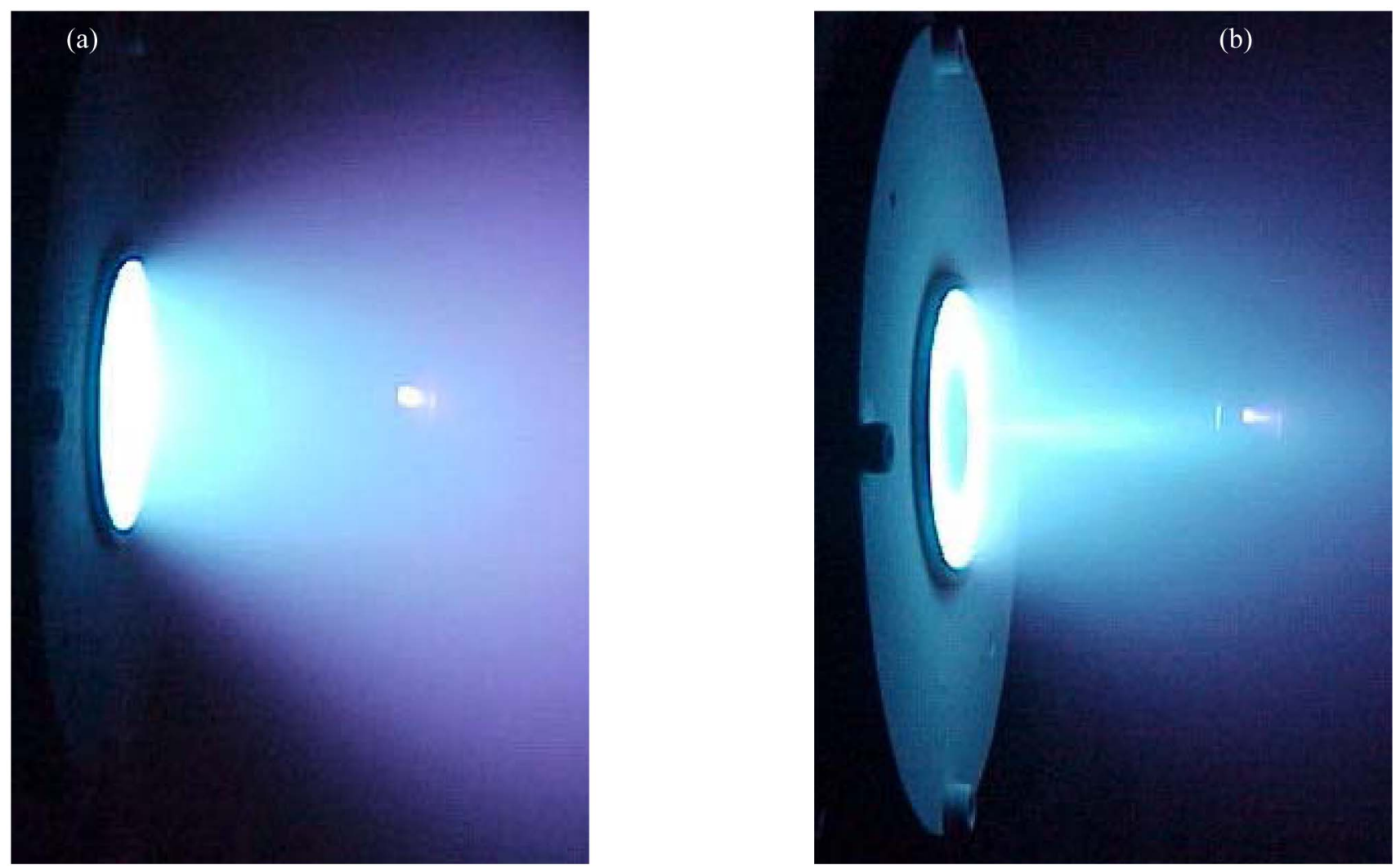

Fig. 1. Plasma plumes of the Hall thruster. (a) Annular Hall thruster. (b) Cylindrical Hall thruster. In both figures, the bright spot to the right is a hollow cathode that emits electrons. The ions flow to the right, and the visible light shown in the figures is mostly from the emission of once-ionized xenon atoms, flowing at about $15 \mathrm{~km} / \mathrm{s}$. The half plume angle of the annular thruster is about $70^{\circ}$, while half plume angle of the cylindrical thruster is about $80^{\circ}$ (which, in the increased cathode current regime, was reduced to about to $\left.50^{\circ}[2]\right)$.

\section{REFERENCES}

[1] A. I. Morozov and V. V. Savelyev, Review of Plasma Physics, vol. 21, B. B. Kadomtsev and V. D. Shafranov, Eds. New York: Consultants Bureau, 2000, p. 203.

[2] Y. Raitses and N. J. Fisch, "Parametric investigations of a nonconventional Hall thruster," Phys. Plasmas, vol. 8, no. 5, pp. 2579-2586, May 2001.

[3] Y. Raitses, L. A. Dorf, A. A. Litvak, and N. J. Fisch, "Plume reduction in segmented electrode Hall thruster," J. Appl. Phys., vol. 88, no. 3, pp. 12631270, Aug. 2000.

[4] N. J. Fisch, Y. Raitses, L. A. Dorf, and A. A. Litvak, "Variable operation of Hall thruster with multiple segmented electrodes," J. Appl. Phys., vol. 89, no. 4, pp. 2040-2046, Feb. 2001.
[5] A. Fruchtman and N. J. Fisch, "Variational principle for optimal accelerated neutralized flow," Phys. Plasmas, vol. 8, no. 1, pp. 56-58, Jan. 2001

[6] A. Fruchtman, N. J. Fisch, and Y. Raitses, "Control of the electric-field profile in the Hall thruster," Phys. Plasmas, vol. 8, no. 3, pp. 1048-1056, Mar. 2001.

[7] A. Fruchtman and A. Cohen-Zur, "Plasma lens and plume divergence in the Hall thruster," Appl. Phys. Lett., vol. 89, no. 11, p. 111 501, Sep. 2006.

[8] A. Smirnov, Y. Raitses, and N. J. Fisch, "Experimental and theoretical studies of cylindrical Hall thrusters," Phys. Plasmas, vol. 14, no. 5, p. 057 106, May 2007.

[9] Y. Raitses, A. Smirnov, and N. J. Fisch, "Enhanced performance of cylindrical Hall thrusters," Appl. Phys. Lett., vol. 90, no. 22, p. 221 502, May 2007. 